either by programming the step-by-step procedure or by using the statistical packages provided.

Finally, a very important benefit of using OMNITAB in a statistics laboratory is that psychology students are introduced to the computer center. Students not only are aided in learning statistics: their experience also dispels many misconceptions of what a computer does, and it provides some basis for understanding how computers affect their lives.

\section{REFERENCES}

Harris, S. $L^{+}, T$. O.MNITAB II, version 5.0: $L^{\dagger}$ ser's reference monual supplement. Austin, Texas: University of Texas Computation Center, TPB-132, 1971.

Hays, W. L. Statistics for psychologists. New York: Holt, Rinehart, \& Winston, 1963.

Hays, W. L. Basic statistics. Belmont, California: Brooks/Cole, 1969.

Hogben, D., Peavy, S., \& Vamer, R. OMNITAB II: L'ser's reference manual. (National Bureau of Standards, Technical Note 552). Washington, D.C: U.S. Government Printing Office, 1971 .

\title{
Teaching young children how to use a computer-based Teletype as a "desk calculator"
}

\section{ELIZABETH F. LOFTUS* \\ New School for Social Research. New York, New York 10011}

The first stage in the research reported here was to teach students the mechanics of how to use a computer-based Teletype as a "desk calculator" in the solution of arithmetic problems. In the second stage, we examined a specific use for the desk-calculator program: we presented a series of word problems to the students and analyzed the solutions of these problems to determine the variables related to problem difficulty.

At Stanford University, the Institute for Mathematical Studies in the Social Sciences (IMSSS) has been developing over the last 9 years a working computer-assisted instruction (CAI) system for classroom use. The research reported here is a small part of a continuing investigation on the potential use and value of such systems. This paper summarizes two demonstrations of a new use for CAI systems.

The demonstrations both involve a computer program which was used to teach bright fifth-grade students, in one case, and disadvantaged sixth-grade students, in the other case, how to use a computer-based Teletype as a desk calculator. Assuming that the students had a basic understanding of the four arithmetical operations (addition, subtraction, multiplication, and division), we asked them to tell the computer which operations they wanted the computer to execute. The actual computations, then, were done by the computer itself.

This report is divided into two major sections. In the first section, we describe the mechanics of how students were taught to use a computer-based Teletype as a desk calculator. In the second section, we describe one use for the desk-calculator program: an analysis of problem-solving performance.

*Requests for reprints should be sent to Elizabeth $F$. Loftus, The Graduate Faculty, New School for Social Research, 66 West 12th Street. New York, New York 10011.

\section{THE DESK-CALCULATOR PROGRAM}

The student terminals used in this project were commercially available Teletype machines, connected by private telephone lines to a computer at the Institute. The control functions for the entire system were handled by the PDP-1, a medium-sized computer with a 32,000-word core and a 4,000-word core interchangeable with any of 32 bands of magnetic drum, together with two large IBM-1301 disk files. All input-output devices were processed through a time-sharing syctem. Two high-speed data channels permitted simultaneous computation and servicing of peripheral devices.

Initial instruction on the Teletype consisted of explaining to each student what the program was generally about. Each student was then given assistance in finding the letters to type his name; no student had any trouble learning how to do this. The program began each day by asking the student to type his assigned number and his name. If the student made an error or gave a fictitious name, such as Napoleon, he was asked to try again. If he typed his number and name correctly, the computer consulted his file and began with the item following the last one completed.

A set of instructions presented via computer taught the student how to command the computer to perform operations on given numbers. The complete set of instructions is given in Loftus (1970). Here, we will briefly list and give an example of each of the abbreviated operation names that the student learned in the instruction set. Student entries are underlined.

(1) $X$ is the answer key.

Suppose the student saw on the printout sheet before him:
G 1) 19 
He could indicate that 19 was his answer by typing $1 \mathrm{X}$, which says to the computer "my answer is on line 1." The line number followed by $X$ indicates what line the final answer is on. ${ }^{1}$

(2) A is the abbreviation for ADD.

An example of how a student might use the A rule is:

$\begin{array}{lll}G & \text { 1) } & 36 \\ G & \text { 2) } & 41 \\ 1.2 A & 3) & 77\end{array}$

By typing " $1.2 \mathrm{~A}$," the student tells the computer to add the number on Line 1 to the number on Line 2 . The computer then prints the result of doing the addition operation.

(3) $\mathrm{S}$ is the abbreviation for SUBTRACT.

An example is:

$$
\begin{array}{llr}
G & \text { 1) } & 500 \\
G & 2) & 48 \\
1.2 S & 3) & 452
\end{array}
$$

(4) $M$ is the abbreviation for MULTIPLY.

An example is:

$$
\begin{array}{lrr}
\mathrm{G} & \text { 1) } & 59 \\
\mathrm{G} & \text { 2) } & 4 \\
1.2 \mathrm{M} & 3) & 236
\end{array}
$$

(5) $\mathrm{Q}$ is the abbreviation for DIVIDE. (Q rather than D was used for divide because $D$ was used for something else in the system.)

An example is:

$\begin{array}{llr}G & \text { 1) } & 77 \\ \mathrm{G} & \text { 2) } & 7 \\ 1.2 \mathrm{Q} & 3) & 11\end{array}$

(6) E means ENTER, and is used to enter a number that is not entered by the computer program. (For example, in a problem that asks the student to find the number of days in 8 weeks, the student would be required to enter the number 7 , the number of days in 1 week. The number 8 would be entered by the computer as a "given number.")

An example is:

$$
\begin{array}{ll}
\mathrm{G} & \text { 1) } 8 \\
\mathrm{E} & 2) \underline{7}
\end{array}
$$

The following sequence of interactions between the

student and the computer illustrates how a word problem is solved in this context. Again, student entries are underlined. The computer first types out the problem, and then types out the numbers in that problem. The student sees on the printout sheet before him:

At the tree nursery, Tom counted 28 rows of pine trees. The forester said that there were 575 trees in each row. How many trees were there at the nursery ...

$$
\begin{array}{llr}
G & \text { 1) } & 28 \\
G & \text { 2) } & 575
\end{array}
$$

At this point, the student tells the computer the operation he wants the computer to perform, and the line numbers to which the operation should apply. For this problem, the student typically types out "1.2M," meaning "multiply the number on Line 1 by the number on Line 2." The computer responds by typing the result of applying the operation, or by typing an error message if the operation could not be applied validly.

The student is still not finished with the problem. He must also indicate where his final answer is by typing the line number on which the answer appears followed by an $X$. The complete protocol for a correct response in the above example, then might be:

At the tree nursery, Tom counted 28 rows of pine trees. The forester said that there were 575 trees in each row. How many trees were there at the nursery . . .

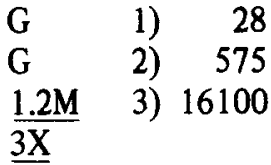

Correct

If the answer is incorrect, "answer is wrong" appears in place of "correct." If the student has not yet indicated his final answer by using " $X$," and if he asks the computer to perform an operation that cannot be applied validly, he receives an error message. In the above example, if instead of typing " $1.2 \mathrm{M}$ " the student had typed "1.2MT," the computer would respond by typing "There is no rule name "MT." "If the student had erroneously typed " 1.2 ," the computer would respond by typing "No rule name given." A flow chart of the program logic is shown in Fig. 1.

A given word problem can often be solved in many ways. The student's own experience and ingenuity determines which rule he uses and what strategy he takes. The computer allows any valid step, regardless of whether it helps reach the solution. Any combination of steps reaching a solution, valid within the rules, is entirely acceptable. A problem such as the following could be solved in several ways:

For an experiment, Susan mixed 7 ounces of glycerin and 14 ounces of alcohol with some water. The resulting mixture contained 45 ounces. How many ounces of water were used? 


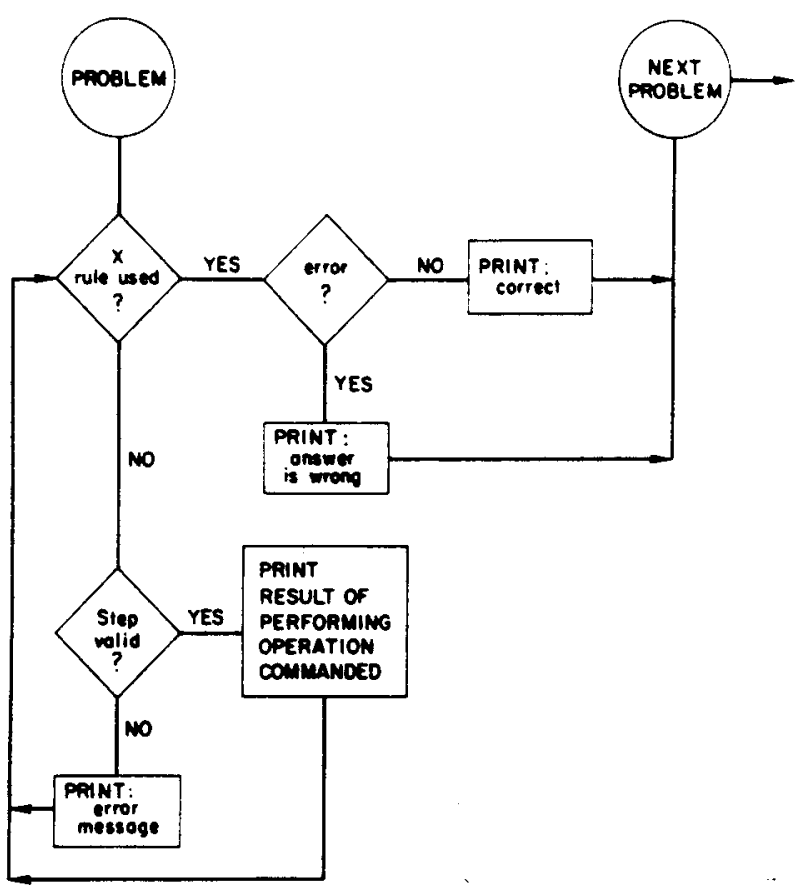

Fig. 1. Flow chart of the program logic for presentation of problems and classification of responses.

It could be solved:

$$
45-(7+14) \text { or }(45-7)-14 \text {. }
$$

A more idiosyncratic solution, such as $45-(7 \times 3)$, is equally acceptable.

In the instruction set, students solved easier problems before being presented with more difficult ones. On several of the problems, the student was invited to ask for a hint after a certain time lapse by the message, "Type $\mathrm{H}$ and a space if you want a hint." If the student asked for a hint on the problem "What is $(486+390)+$ 707?" he was told "First find $486+390$. Then add the sum to 707." No hints were available on multiple-choice problems; the student had to guess until he got the problem correct.

While the student was trying to reach a solution, the computer did four things. (1) Every student command was examined to see if it was a valid step and if the syntax was correct. If incorrect, the computer printed out an error message. (2) If valid, the computer performed whatever step the student commanded. (3) The desired solution was compared to the final answer indicated by "X." If the two were identical, the computer terminated the problem after typing "correct." If they were not identical, the computer typed "answer is wrong." (4) After a fixed time lapse. the computer offered a hint on certain problems.

\section{ANALYSIS OF PROBLEM-SOLVING PERFORMANCE}

There are numerous purposes to which such a desk-calculator program could be put. Since our primary interest was in the analysis of problem-solving performance, we followed the initial instructional program with a series of arithmetic word problems. The students solved these word problems using the rules they had learned in the instruction set. We then analyzed their solutions to determine the variables related to problem difficulty, in an attempt to understand what variables cause some word problems to be hard to solve while others are easy. In this section, we first briefly describe the set of problems; second, we present the results of our analyses of those problems; and third, we discuss some implications of these results.

\section{Fifth-Grade Students}

\section{The Word-Problem Set}

Because the fifth-grade students were from an accelerated group, we presented them with 68 word problems designed to be of appropriate difficulty for sixth-grade students. The students used the rules they learned in the instruction set to solve these problems. As was done in the initial instruction set, the computer, after typing out each problem, typed out all of the numbers given in the problem as "given numbers." The student then, in a series of commands, told the computer what to do with these numbers. After each student command, the computer typed out the result of the operation the student had commanded the computer to perform. The student indicated his final answer by using the " $\mathrm{X}$ " key. If the final answer was correct, the computer typed the message "correct" and went on to the next problem. If the final answer was immerect, the computer typed "answer is wrong" and went to the next problem.

The students were not allowed to use pencil or paper when working on the Teletype. Each exercise was worked on the machine, so that all responses could be recorded.

The student was signed off, as during the instruction set, with a "goodbye" message, and "please tear off on the dotted line." The study with fifth-grade students is reported more extensively in Suppes, Loftus, and Jerman (1969).

\section{Sixth-Grade Students}

For the sixth-grade students, 100 word problems, designed to be of appropriate difficulty for sixth-grade students, were used. The procedure for presenting these problems was similar to that described above, and is reported more fully in Loftus (1970).

\section{Analysis of Word Problem Solutions Fifth-Grade Students}

To reiterate, our primary interest was to investigate the variables that are related to problem difficulty. The variables $^{2}$ we investigated were: (1) Operations-This refers to the minimum number of different operations required to reach the correct solution. (2) Steps-This refers to the minimum number of steps required to reach 
the correct solution. ${ }^{3}$ (3) Length-This refers to the number of words in the problem. (4) Sequence-This refers to whether or not the problem can be solved by the same operation(s) as the problem that preceded it. (5) Verbal clue-This refers to whether the problem contains a verbal clue to the operation(s) required to solve the problem. (6) Conversion-This refers to whether conversion of units, such as from months to weeks, is required to solve the problem.

In order to determine the predictive worth of the six variables, we first computed the probability of a correct response for each word problem. Next, we obtained regression coefficients for each of the six variables. A multiple linear regression analysis program, adapted for the PDP-1 computer at Stanford, was used to obtain regression coefficients. multiple correlation $R$, and $R^{2}$. The square of the multiple correlation coefficient. $R^{2}$. is an estimate of the amount of variance accounted for by the regression model. In this case, $45 \%$ of the variance in probability of a correct response is accounted for by six variables described above. The sequence variable was the most important of the six variables. Thus, a problem was easy to solve if it could be solved by the same operation(s) as the problem that preceded it. Conversion and operations were also valuable predictors of the probability of a correct response for each item. Thus, these bright fifth-grade students were more likely to solve a problem correctly if: (1) it was similar to the problem that preceded it, (2) its solution required a small number of different operations, and (3) it did not isquire a cenversion of units.

\section{Sixth-Grade Students}

The solutions of word problems solved by these students were analyzed in terms of the same six variables described above, plus two others. The additional variables were: (1) Order-This refers to whether the numerical data are presented in some order other than an order in which they could be used to solve the problem. (2) Depth-This is an index of the structural complexity of the word problem from a linguistic point of view. It is described more fully in Loftus (1970).

Again. our main task was to report the worth of the eight variables for successfully predicting the probability of a correct response for each word problem. The regression coefficients. multiple correlation $R$, and $R^{2}$ were obtained from a stepwise. multiple linear regression analysis program. BMD 02R, adapted for Stanford University's IBM 360 computer. $\mathrm{R}^{2}$. which is an estimate of the amount of variance accounted for by the eight variables, was 70 .

Again. the sequence variable was the most important of the eight variables. Operations. depth. length. and conversion were also significant predictors of the probability of a correct response for each item. Thus. a sixth-grade student was more likely to solve a problem correctly if (1) it could be solved by the same operation(s) as the problem that preceded it. (2) its solution required a small number of different operations, (3) it was linguistically less complex, (4) it had a small number of words, and (5) it did not require a conversion of units.

\section{Implications}

In this section, we have summarized our analyses of the response performance of children who solved arithmetic word problems. With a few variables, we have brought a considerable amount of order to a large problem set which at first glance appears to be quite complex. We have determined that sequence, operations, depth, length, and conversion are significant determinants of word problem difficulty. In view of the intrinsic complexity of word problem solving, we have accounted for an impressive proportion of the variance in such behavior.

A comparison of the bright fifth-grade students and disadvantaged sixth graders is interesting and potentially instructive. The most suggestive finding regarding these two groups is the importance of the sequence and operations variables. For the bright as well as for the disadvantaged students, these two variables are highly significant determinants of problem solving difficulty. Whether students are bright or dull, they are more likely to solve a problem correctly if it is similar to the problem that preceded it or if its solution requires a small number of different operations. The implication is that many aspects of the internal processing done by students when they solve problems do not differ for children differing in mental ability.

One finding regarding the conversion variable is also interesting. It happens that the disadvantaged group of sixth graders actually included students from two elementary schools. call them School A and School B. In School A. $82 \%$ of the children are black and the average sixth-grade IQ is 93. In School B, 59\% of the children are black and the average sixth-grade IQ is 99 . When multiple regression analyses were performed on the two groups taken separately. it was found that the conversion variable was not an important predictor for the School A $(\mathrm{IQ}=93)$ students, but was reasonably important for School B (IQ =99) students. For the bright fifth-grade students (IQ $=120)$, the conversion variable was the second-most important variable in terms of predictive worth. It appears that there may be some relationship between mental ability and the importance of the conversion variable. No attempt has been made to estimate parameters that would reflect mental ability. or to explore the relationship between mental ability and the conversion variable, because accounting for differential mental ability is beyond the scope of this research.

The results obtained in this study give a clear indication of the difficulty in constructing an explanatory theory that is adequate to account for all the difficulties students encounter in solving word problems. We have just soratched the surface of the 
complete syntactic and semantic analysis that will be required to predict all the details that must be accounted for in the behavior of students. The present effort has been an attempt to understand what variables cause some word problems to be hard to solve while others are easy. Only after we have a grip on these variables can we hope to be able to formulate a coherent theory of problem solving. Thus, these analyses represent tentative, preliminary, first steps toward the construction of a more mature theory. Further development of such a theory, as well as any discussion of the implications of the various predictive analyses for the teaching of problem solving, must await much needed additional research. In particular, more refined analysis with data from larger numbers of students is needed. We are convinced that deeper investigations in this direction are essential to a better understanding of problem solving.

\section{REFERENCES}

Loftus, E. J. F. An analysis of the structural variables that determine problem solving difficulty on a computer-based Teletype. Technical Report No. 162, December 18, 1970 Stanford University, Institute for Mathematical Studies in the Social Sciences.

Suppes, P.. Loftus, E. F., \& Jerman, M. Problem-solving on a computer-based Teletype. Educational Studies in Mathematics, 1969, 2, 1-15.

\section{NOTES}

1. "G" stands for "given number." Whenever a student was given a word problem to solve, all the numbers in the problem were typed out as given numbers just after the word problem itself was typed out. The reason for designing the program in this way was to reduce the time required for students to input large numbers themselves. Requiring students to input very large numbers slows down learning and can be relatively demanding. especially during early stages of learning. Instead, we asked the student to input on the keyboard what rule he wished to apply to what given numbers. All he had to input, then, was the rule and the lines to which that rule was to be applied.

2. The rationale for these variables is discussed in Suppes Loftus, and Jerman (1969).

3. The operations variable and the steps variable may be distinguished more clearly if we consider a problem which requires two or more computational processes before the answer can be found. A problem that asks the student to find the average of 10 numbers would require 10 separate steps but only two different operations. 